\title{
Mini-commentary on BJOG-20-0442.R2
}

\author{
Sania Latif ${ }^{1}$ and Dimitrios Mavrelos ${ }^{1}$ \\ ${ }^{1}$ University College London Hospitals NHS Foundation Trust
}

September 11, 2020

Mini-commentary on BJOG-20-0442.R2: Impact of hysterectomy on analgesic, psychoactive and neuroactive drug use in women with endometriosis: nationwide cohort study

\section{Endometriosis-associated pain in women undergoing hysterectomy}

Sania Latif, ${ }^{1}$ Dimitrios Mavrelos ${ }^{1}$

1) University College London Hospitals NHS Foundation Trust

25 Grafton Way

London, UK NW1 2PG

Pain is a cardinal symptom of endometriosis and its management can be challenging. Analgesics are commonly used as first line treatment for endometriosis-associated pain, despite there being a paucity of evidence regarding their use for this indication. Clinicians are advised to consider NSAIDs or other analgesics to reduced endometriosis-associated pain due to the known benefit of NSAIDs in primary dysmenorrhea (ESHRE guideline "Management of women with endometriosis." Dunselman et al.,Hum Reprod. 2014; 29(3):400-12). Women who fail to respond to conservative treatments and who have completed their family may be offered hysterectomy with removal of the ovaries and all visible endometriosis lesions, alongside advice that hysterectomy will not necessarily cure the symptoms or the disease.

Brunes et al. (BJOG 2020; https://doi.org/10.1111/1471-0528.16469) analysed data from two Swedish population-based registers: the Swedish National Quality Register of Gynaecological Surgery (GynOp) and the Swedish National Drug Register. Data was collected prospectively and reported by both patient and surgeon in this nationwide cohort study. Brunes et al. (BJOG2020) reported that women with endometriosis undergoing hysterectomy have a higher prescription rate of analgesics. The prescription of analgesics, psychoactive drugs and neuroactive drugs did not decrease when they compared drug use three years preoperatively with that 3 years post-operatively.

Hysterectomy for chronic non-specified pelvic pain associated with endometriosis is a successful approach in many women, but some women do not obtain any relief of pain after hysterectomy (Martin. J Minim Invasive Gynecol, 2006; 13:566-572). It is likely that this is because there are difficulties in evaluating hysterectomy for endometriosis-associated pain and in establishing whether endometriosis is the cause of pain or a coincidental finding in a woman with chronic pelvic pain. Other factors impacting the success of surgery and the level of post-operative analgesic use include whether there is effective removal of endometriotic lesions or whether the ovaries are removed. Hysterectomy with ovarian conservation has been reported to have a 6 -fold risk for development of recurrent pain, with a recurrence rate of $62 \%$ in advanced stage endometriosis (Martin. J Minim Invasive Gynecol, 2006; 13:566-572). Surgical effort should always aim to eradicate the endometriotic lesions completely.

Clinicians and patients should be aware that the expected benefit of surgery is operator dependent and that the extent and duration of therapeutic benefit of surgery are poorly defined (Vercellini et al.Hum Reprod 
Update. 2009;15(2):177-188). There is a need for prospective clinical trials and long-term follow up studies in women with endometriosis to compare treatment options including hysterectomy for endometriosis-associated pain using defined outcome measures.

Disclosure of interests: None declared. Completed disclosure of interest forms are available to view online as supporting information. 